other organs ${ }^{5,6}$. The data suggested that one of the risk factors endemic to these areas, aflatoxin $B_{1}$ or hepatitis $B$ virus (HBV), was responsible for the mutations. However, it was possible that the mutations were not mutagen-specific but rather were tumour-type specific, so that HCC from regions without these risk factors might also contain the same mutations.

This alternative explanation is made less likely by the new data from Ozturk and collaborators ${ }^{1}$, which show that HCC from low-aflatoxin exposure areas (even those with a high HBV infection rate) only rarely contain the $249^{\text {ser }}$ mutation. Combined with the fact that aflatoxin $B_{1}$ is known to induce $G \rightarrow T$ transversions in simple model systems, the most likely interpretation of the data is that aflatoxin $B_{1}$, in the context of chronic viral hepatitis, drives HCC in humans by forming deoxyguanosine adducts at codon 249 , resulting in substitutions of $\mathrm{T}$ for $\mathrm{G}$.

Another strong case is made by Brash et $\mathrm{al}^{2}{ }^{2}$ for the involvement of $\mathrm{p} 53$ mutation in human squamous cell carcinomas (SCC) of the skin. Over half of the invasive SCC studied had p53 gene mutations, all of the mutations being at dipyrimidine sites (such a predominance is characteristic of UV light-induced mutations in model systems). Moreover, a quarter of the mutations resulted in $\mathrm{CC}$ to $\mathrm{TT}$ double-base changes. These double-base changes are virtually pathognomonic of a UV-based aetiology and have rarely been observed in internal tumours.

The analysis of p53 in other tumour types is also proving to be revealing. Lung and oesophageal cancers frequently contain base-pair changes characteristic of those produced by the mutagens present in tobacco smoke and alcohol, respectively ${ }^{7,8}$. These agents are known to be risk factors from epidemiological studies. In the future, we might expect somatic mutations to point towards specific aetiological factors in cases where epidemiologists are unable to pinpoint specific agents.

Many questions about the relationship between environmental mutagens and cancer remain, even for HCC and SCC. Cancer apparently arises through the sequential accumulation of mutations in several oncogenes and/or suppressor genes $^{9}$. Both HCC and SCC probably contain at least four or five such mutations in each tumour. Are all these mutations induced by aflatoxin $B_{1}$ (or sunlight) or do these agents simply place the final straw on the camel's back? This question will become tractable when more mutant genes in these tumours are identified.

The data also have implications for a debate about the nature of human carci- nogenesis. It has recently been argued (1) that human exposure to industrially derived carcinogens is minuscule compared to that derived from exposure to naturally derived carcinogens, and (2) that carcinogens can act by direct adduct-driven mutagenesis and/or by causing increased cellular turnover (mitogenesis) which indirectly increases mutation rates ${ }^{10}$. The new reports bear on these arguments in several ways. Although some people are convinced that technology is responsible for most of society's ills, these first clear examples of carcinogen-induced mutations in human cancer point towards natural rather than man-made agents. Second, the base-pair changes in SCC and HCC p53 genes strongly suggest that they are the result of adduct-driven mutagenesis, rather than secondary effects of increased mitogenesis (although the results cannot rule out another role for carcinogen-induced mitogenesis in the development of these tumours). Third, one might ask why, if the dose of naturally derived mutagens (either from normal oxidative metabolism or from chemicals endogenously present in foods) is so high, is the additional exposure to single mutagens such as aflatoxin $B_{1}$ and sunlight rate-limiting for cancer development?

This new molecular epidemiology also provides lessons for cancer prevention. It is one thing to tell a tanning champion from California (or a regulatory official in Asia) that epidemiological and animal evidence suggest certain preventative measures. It is quite another to say we know that light of $300 \mathrm{~nm}$ induces mutations at pyrimidine dimers in skin-cell p53 genes which will prevent that gene from suppressing tumour growth. The latter is more compelling because of its clarity and inherent logic, providing a coherent picture of how a specific agent contributes to the development of human cancer in molecular detail. The potential economic and human benefits of putting this new knowledge into practice, for prevention of cancer, are immense.

Bert Vogelstein and Kenneth W. Kinzler are at the Johns Hopkins Oncology Center, 424 North Bond Street, Baltimore, Maryland 21231, USA.

1. Ozturk, M. and collaborators Lancet 338, 1356-1359 (1991).

2. Brash. D. E. et al. Proc. natn. Acad. Sci. U.S.A. 88, 10124-10128 (1991).

3. Barbacid, M. A. Rev. Biochem. 56, 779-827 (1987).

4. Bos, J. L. Cancer Res. 49, 4682-4689 (1989).

5. Hsu, I. C. et al. Nature 350, 427-428 (1991).

6. Bressac, B., Kew, M., Wands, J. \& Ozturk, M. Nature 350, 429-431 (1991).

7. Hollstein, M., Sidransky, D., Vogelstein, B. \& Harris, C. C. Science 253, 49-53 (1991)

8. Bennett, W. P. et al. Oncogene 6, 1779-1784 (1991).

9. Fearon, E. R. \& Vogelstein, B. Cell 61, 759-767 (1990).

10. Ames, B. N. \& Gold, L. S. Science 249, 970-971 (1990).

\section{Noses have ears}

THE smaller the animal, the higher the range of sound frequencies that it can generate and hear. Elephants have an upper limit of hearing well below the human one of $15 \mathrm{kHz}$ or so; cats can hear up to $50 \mathrm{kHz}$ and bats up to $120 \mathrm{kHz}$. All this, says Daedalus, makes good physical sense. Smaller animals have smaller ears and voice boxes, whose smaller resonant structures create and respond to higher frequencies. Extending the argument, Daedalus expects insects to communicate in the 1-MHz band. Modern ultrasonic technology can easily generate and detect frequencies around $1 \mathrm{MHz}$, even though in air they travel only short distances. Already ultrasonic generators are sold to discourage cockroaches and similar pests. This simplistic technology could easily be developed. By detecting, decoding and imitating the ultrasonic communications of ants, hornets and other social insects, specific messages could be directed at them. They could be directed not to seek food in our buildings, and to make their nests away from them.

Bacteria, much smaller even than insects, should communicate at $1 \mathbf{G H z}$ and above. This sonic region encroaches into the range of molecular rotational and even vibrational frequencies. According to one theory, our sense of smell works by detecting such molecular vibrations. Clearly, says Daedalus, at these high frequencies the distinction between an ear and a nose disappears. A bacterium must carry on its surface a range of sensory molecules or molecular clusters which respond to specific molecules nearby, either by direct vibrational contact, or by detecting sonically radiated molecular vibrational modes.

So Daedalus is devising non-contact toxins to kill or control bacteria. They never even touch the target microorganism, but merely radiate the appropriate molecular frequencies. Such a toxin might, for example, imitate the vibrational 'smell' of a nutritional molecule. The organism would open its cell wall in anticipation, and leak to death. Daedalus is vacuum-coating fine particles of bacterial poisons like phenol and penicillin, with very thin layers of gold, magnesium fluoride and so on. Such coatings have few vibrational modes of their own, but should faithfully transmit those of the molecules within. If these coated particles turn out to damage or influence bacteria, the way will be open for a whole new range of microencapsulated drugs. Ferociously reactive, they will withstand body chemistry and exert no damaging side effects, because they will never escape from their vibrotransparent envelopes.

David Jones 